\title{
Statistics of normal mode amplitudes in an ocean with random sound-speed perturbations: Cross-mode coherence and mean intensity
}

\author{
John A. Colosi \\ Department of Oceanography, Naval Postgraduate School, Monterey, California 93943 \\ Andrey K. Morozov \\ Department of Applied Ocean Physics and Engineering, Woods Hole Oceanographic Institution, Woods \\ Hole, Massachusetts 02543
}

(Received 20 November 2008; revised 13 May 2009; accepted 14 May 2009)

\begin{abstract}
In this paper Creamer's [(1996). J. Acoust. Soc. Am. 99, 2825-2838] transport equation for the mode amplitude coherence matrix resulting from coupled mode propagation through random fields of internal waves is examined in more detail. It is shown that the mode energy equations are approximately independent of the cross mode coherences, and that cross mode coherences and mode energy can evolve over very similar range scales. The decay of cross mode coherence depends on the relative mode phase randomization caused by coupling and adiabatic effects, each of which can be quantified by the theory. This behavior has a dramatic effect on the acoustic field second moments like mean intensity. Comparing estimates of the coherence matrix and mean intensity from Monte Carlo simulation, and the transport equations, good agreement is demonstrated for a $100-\mathrm{Hz}$ deep-water example. (C) 2009 Acoustical Society of America. [DOI: 10.1121/1.3158818]
\end{abstract}

PACS number(s): 43.30.Bp, 43.30.Re, 43.60.Cg [WLS]

Pages: $1026-1035$

\section{INTRODUCTION}

Numerous observations and numerical studies have shown that random internal-wave-induced sound-speed perturbations in both shallow- and deep-water environments can cause significant changes in the mean acoustical intensity relative to the unperturbed intensity. Examples in deep-water problems are the depth broadening of the acoustic finale (Worcester et al., 1994; Colosi et al., 1994; Colosi and Flatté, 1996; Worcester et al., 1999) and the so-called deep shadow zone arrivals (Dushaw et al., 1999; Flatté and Colosi, 2008; Van Uffelen et al., 2009). For shallow-water problems, on the other hand, the acoustic arrivals are seen to have significant time spreading (Tielburger et al., 1997; Fredricks et al., 2005) which could be due to both random linear internal waves and nonlinear internal solitary waves. Lacking, however, has been a theoretical understanding of the dominant acoustic scattering physics leading to the mean redistribution of the acoustical energy. This theoretical understanding is prerequisite to formulating a useful reduced physics model to predict such effects without having to resort to time consuming Monte Carlo simulations. Available theoretical models to understand this behavior have fallen short: Path integral results have been shown to break down at long range due to the instability of the unperturbed ray path (Colosi et al., 1999; Beron-Vera et al., 2003), and ray chaos methods cannot accurately predict intensity. Coupled mode approaches are thus promising. The seminal work of Creamer (1996) first introduced a transport equation for the cross mode coherence matrix, a necessary ingredient for the calculation of second moments like mean intensity. However in that work, the coherence terms were neglected in order to study the asymptotic evolution of mode energy, the diagonal of the coherence matrix. Later work by Voronovich and Ostashev $(2006,2009)$ has refocused on the coherence matrix and has included out-of-plane coupling effects to predict horizontal coherence.

As a first step toward gaining further physical understanding of the aforementioned observed intensity, theoretical results for the mean intensity at a single frequency are presented in this paper utilizing the simple two-dimensional (2D) results of Creamer (1996). The normal mode framework developed by Dozier and Tappert (1978a, 1978b) and extended by Dozier (1983) and Creamer (1996) takes advantage of the facts that (1) the coupling is weak (i.e., there is small angle forward scattering) and (2) that the Markov approximation is valid. Within this framework, this paper delves somewhat more deeply into the transport equation for the cross mode coherence matrix to underscore some points not previously appreciated. In particular, it is shown that the decay of cross mode coherences is controlled by terms in the equations associated with adiabatic and mode coupling induced phase randomization. In the deep-water cases addressed here, the coupling effects dominate, and thus mode energy and cross mode coherence evolve over similar range scales; a result contrary to conventional speculation in the literature (Dozier and Tappert, 1978a, 1978b; Creamer, 1996). Second it is shown that the evolution equation for the modal energies is insensitive to the actual values of the offdiagonal terms or cross mode coherences. This result explains the successful predictions of modal energies using theory which neglects cross mode coherence terms (Dozier and Tappert, 1978a, 1978b; Creamer, 1996). Finally using Monte Carlo simulation techniques for a deep-water example 
the cross mode coherence matrix evolution equations are shown to produce accurate predictions of mean intensity out to very long range.

The outline of this paper is as follows. Section II describes the evolution equation for the cross mode coherence matrix. Section III addresses the mode energy observable and its connection to the off-diagonal cross mode coherences. Section IV gives a two mode example which helps understand the nature of the solutions to the evolution equation, while Sec. V demonstrates the accuracy of the method for predicting mean intensity. Section VI gives brief summary and conclusions.

\section{2D COUPLED MODE THEORY}

The acoustic pressure at frequency $\omega$ is expressed in terms of the normal mode expansion (Creamer, 1996)

$$
p(r, z ; \omega)=\sum_{n=1}^{N} \frac{a_{n}(r) \phi_{n}(z)}{\sqrt{k_{n} r}},
$$

where the unperturbed normal mode equation, $\rho_{0}(z) \partial / \partial z\left(\rho_{0}^{-1}(z) \partial \phi_{n} / \partial z\right)+\left(\bar{k}^{2}(z)-k_{n}^{2}\right) \phi_{n}=0$, gives the eigenmodes $\phi_{n}$ and eigenwavenumbers $k_{n}$, and all the variability is contained in the mode amplitude $a_{n}$. Here the background density is $\rho_{0}(z)$, and sound speed is $c(r, z)=\bar{c}(z)+\delta c(r, z)$, with $\bar{k}(z)=\omega / \bar{c}(z)$. Without any loss of generality the modal wavenumber can be considered to have a small complex component from attenuation such that $l_{n}=k_{n}+i \alpha_{n}$. In addition it is useful to define a reduced modal amplitude quantity that removes the rapid oscillations in range so that $\psi_{n}(r)$ $=a_{n}(r) e^{-i l_{n} r}$ with the result that the mean intensity for weak attenuation is given by

$$
\begin{aligned}
\langle I(r, z)\rangle & =\left\langle|p(r, z)|^{2}\right\rangle \\
& =\sum_{n=1}^{N} \sum_{p=1}^{N}\left\langle\psi_{n} \psi_{p}^{*}(r)\right\rangle \frac{e^{i\left(l_{n}-l_{p}^{*}\right) r}}{r} \frac{\phi_{n}(z) \phi_{p}(z)}{\sqrt{k_{n} k_{p}}},
\end{aligned}
$$

where $\left\langle\psi_{n} \psi_{p}^{*}(r)\right\rangle$ is the cross mode coherence matrix. Here the importance of the cross mode coherences to the mean intensity observable is clearly evident. Figure 1 shows example calculations of deep-water $100-\mathrm{Hz}$ directed beam propagation with and without ocean internal-wave-induced sound-speed perturbations (for simulation details see the Appendix). The spatial coherence of the perturbed and mean intensity beams in depth and range for the first $500-1000 \mathrm{~km}$ shows visually that the cross mode coherence is not decaying rapidly with range. After 1000-km range a smooth nearly featureless mean intensity pattern is seen to be due to an absence of cross mode coherence. Morozov and Colosi $(2005,2007)$ showed other numerical examples of $100-, 125-$, and $250-\mathrm{Hz}$ directed beam propagation through ocean internal waves in a deep-water environment which have a similar character. It should be noted that some simple analytic estimates describing cross mode coherences have been derived using the ray-mode duality (Virovlyanskii, 1989; Virovlyanskii et al., 1989).

Dozier (1983) and Creamer (1996) showed that the coupled mode equations for small attenuation are

$$
\frac{d \psi_{n}}{d r}=-i \sum_{m=1}^{N} \rho_{m n}(r) e^{i l_{m n} r} \psi_{m}(r),
$$

where $l_{m n}=l_{m}-l_{n}=k_{m n}+i \alpha_{m n}$, and the symmetric coupling matrix $\rho_{m n}(r)$ is given by

$$
\rho_{m n}(r)=\frac{k_{0}^{2}}{\sqrt{k_{n} k_{m}}} \int_{0}^{D} \frac{\phi_{n}(z) \phi_{m}(z)}{\rho_{0}(z)} \mu(r, z) d z .
$$

Here $k_{0}$ is a reference wavenumber, $D$ is the water depth, and $\mu(r, z)=\delta c(r, z) / c_{0}$ is the random fractional sound-speed fluctuation, assumed zero in the seabed. This equation accurately models wide angle propagation in the sound channel.

\section{A. Correlation function of the coupling matrix}

The correlation function of the coupling matrix will be central to this analysis. Writing $\mu(z, r)$ in terms of a linear superposition of internal waves with mode number $j$ and Cartesian horizontal wavenumber $k_{r}$, the coupling matrix becomes

$$
\begin{aligned}
\rho_{m n}(r)= & \frac{\mu_{0} k_{0}^{2}}{\sqrt{k_{m} k_{n}}} \int_{0}^{D} d z\left(\frac{N(z)}{N_{0}}\right)^{3 / 2} \frac{\phi_{n}(z) \phi_{m}(z)}{\rho_{0}(z)} \\
& \times \sum_{j=1}^{\infty} h_{j} \sin [\pi j \hat{z}(z)] \int_{-\infty}^{\infty} d k_{r} b_{j}\left(k_{r}\right) e^{i k_{r} r},
\end{aligned}
$$

where $N(z)$ is the buoyancy frequency profile, $\mu_{0}$ is a reference rms fractional sound speed, $h_{j}$ and $b_{j}\left(k_{r}\right)$ are complex Gaussian random variables, and $\hat{z}(z)$ is the Wentzel-KramersBrillouin-Jeffreys (WKBJ) stretched vertical coordinate (Colosi and Brown, 1998). Multiplying by $\rho_{q p}^{*}(r-\xi)$ and taking the expectation value the result is

$$
\begin{aligned}
\Delta_{m n, q p}(\xi) & =\left\langle\rho_{m n}(r) \rho_{q p}^{*}(r-\xi)\right\rangle \\
& \left.=\sum_{j=1}^{\infty}\left\langle\left|h_{j}\right|^{2}\right\rangle G_{m n}(j) G_{q p}(j) \int_{-\infty}^{\infty} d k_{r} r\left|b_{j}\left(k_{r}\right)\right|^{2}\right\rangle e^{-i k_{r} \xi},
\end{aligned}
$$

where

$$
\begin{aligned}
G_{m n}(j)= & \mu_{0} k_{0}^{2} \sqrt{\frac{2}{k_{n} k_{m}}} \int_{0}^{D} d z\left(\frac{N(z)}{N_{0}}\right)^{3 / 2} \\
& \times \sin [\pi j \hat{z}(z)] \frac{\phi_{n}(z) \phi_{m}(z)}{\rho_{0}(z)},
\end{aligned}
$$

and from the Garrett-Munk (GM) spectrum the expressions are

$$
\left\langle\left|h_{j}\right|^{2}\right\rangle=\frac{1}{M_{j}} \frac{1}{j^{2}+j_{*}^{2}},
$$



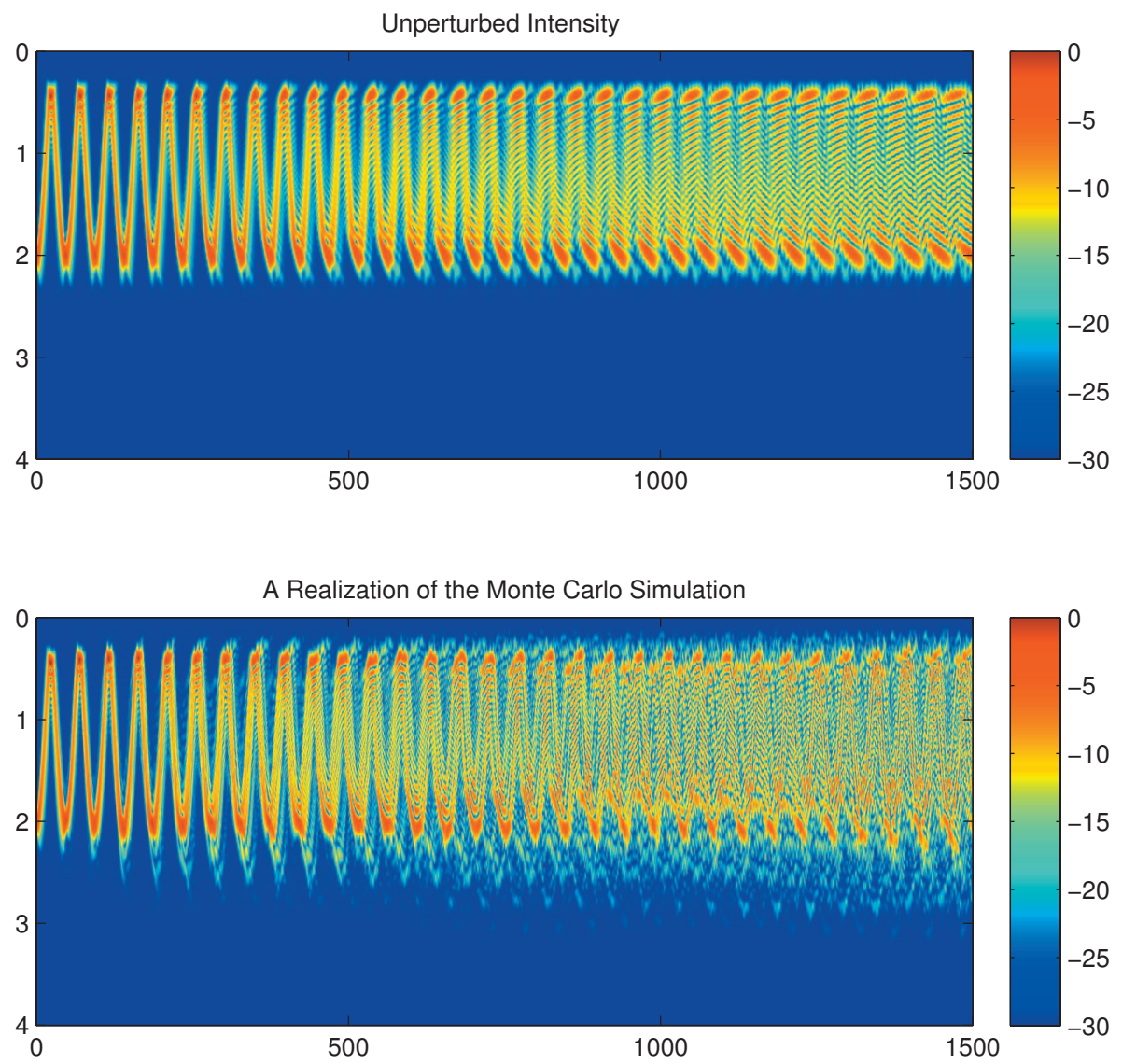

FIG. 1. Acoustic beams from 100-Hz numerical simulations using the canonical ocean described in the Appendix. The upper panel displays the unperturbed acoustical beam while the middle panel shows a realization of the beam propagation through a random realization of internal-waveinduced sound-speed perturbations. The lower panel shows the mean intensity averaged over 500 realizations of the internal wave field. Cylindrical spreading is not included in the mean intensity. The intensity scale is decibel referenced to the maximum value.

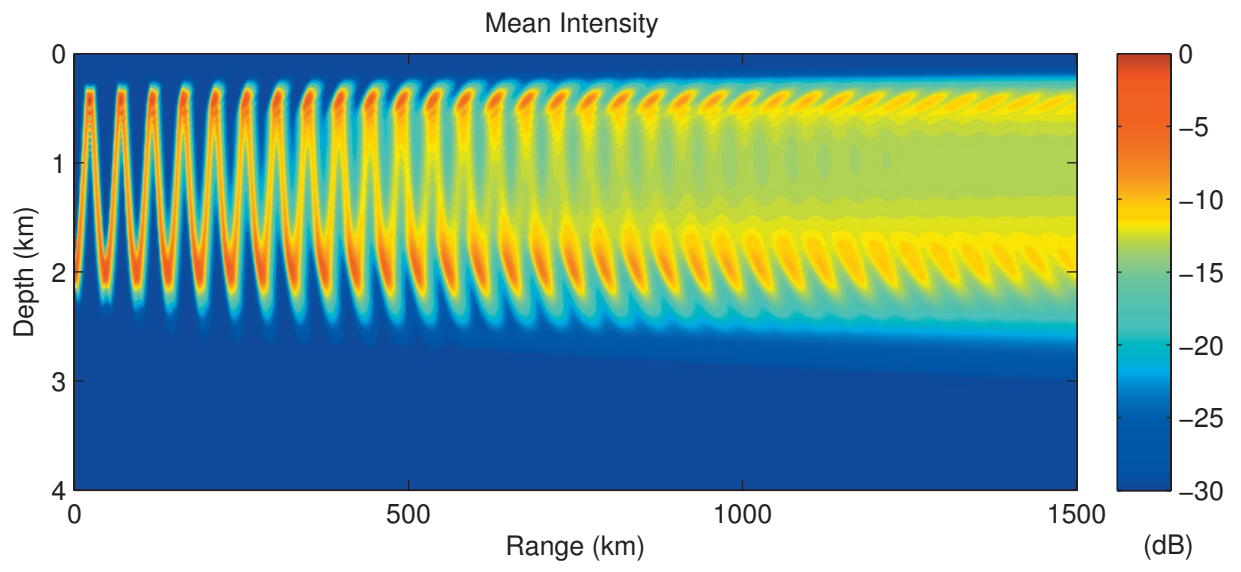

$$
\begin{aligned}
\left\langle\left|b_{j}\left(k_{r}\right)\right|^{2}\right\rangle= & \frac{2}{\pi^{2}}\left[\frac{k_{j}}{k_{r}^{2}+k_{j}^{2}}\right. \\
& \left.+\frac{1}{2} \frac{k_{r}^{2}}{\left(k_{r}^{2}+k_{j}^{2}\right)^{3 / 2}} \log \left(\frac{\left(k_{r}^{2}+k_{j}^{2}\right)^{1 / 2}+k_{j}}{\left(k_{r}^{2}+k_{j}^{2}\right)^{1 / 2}-k_{j}}\right)\right] \\
\simeq & \frac{1}{\pi} \frac{\hat{k}_{j}}{\hat{k}_{j}^{2}+k_{r}^{2}},
\end{aligned}
$$

with $\quad M_{j}^{-1}=\sum_{j=1}^{\infty}\left(j^{2}+j_{*}^{2}\right)^{-1}, \quad k_{j}=\pi f j / N_{0} B, \quad \hat{k}_{j}^{2}=2 k_{j}^{2}, \quad N_{0} B$ $=\int_{0}^{D} N(z) d z$, and $f$ is the Coriolis parameter (Morozov and Colosi, 2007). Using the approximation in the last line of Eq. (8) a useful and accurate approximate form of the correlation function is obtained, namely,

$$
\Delta_{m n, q p}(\xi)=\sum_{j=1}^{\infty}\left\langle\left|h_{j}\right|^{2}\right\rangle G_{m n}(j) G_{q p}(j) e^{-\hat{k}_{j}|\xi|} .
$$

The matrices involved in the cross-mode coherence transport equation involve integrals over the correlation function $\Delta_{m n, q p}$ of the form

$$
I_{m n, q p}=\int_{0}^{\infty} d \xi \Delta_{m n, q p}(\xi) e^{i l_{p q} \xi}
$$

Some useful symmetry properties of Eq. (10) are $I_{m n, q p}$ $=I_{n m, q p}$ and $I_{m n, p q}=I_{m n, q p}^{*}$. For zero attenuation, the real part of this function is closely related to the wavenumber spectrum of the coupling matrix, that is, 


$$
\frac{1}{\pi} \operatorname{Re}\left(I_{m n, q p}\right)=\left\langle\hat{\rho}_{m n} \hat{\rho}_{q p}^{*}\left(k_{p q}\right)\right\rangle=\frac{1}{2 \pi} \int_{-\infty}^{\infty} d \xi \Delta_{m n, q p}(\xi) e^{i k_{p q} \xi} .
$$

This function then is associated with resonance conditions that pick out specific internal wave wavenumbers that contribute to mode coupling. In addition for $q=p$ this function has the important physical interpretation in terms of a correlation length in the horizontal, that is, $L_{H}(m n, p)$ $=I_{m n, p p} / \Delta_{m n, p p}(0)$. When attenuation is added to the picture the resonance is shifted so that different wavenumbers contribute to the coupling (Colosi, 2008). Using the approximate correlation function in Eq. (9), the transport matrix has a useful analytic form

$$
I_{m n, q p}=\sum_{j=1}^{\infty}\left\langle\left|h_{j}\right|^{2}\right\rangle G_{m n}(j) G_{q p}(j) \frac{\hat{k}_{j}+\alpha_{p q}+i k_{p q}}{\left(\hat{k}_{j}+\alpha_{p q}\right)^{2}+k_{p q}^{2}}, \quad\left|\alpha_{p q}\right|<\hat{k}_{j},
$$

where the attenuation shifting of the resonance is evident.

\section{B. Cross-mode coherence transport equation: A heuristic derivation}

Creamer's (1996) transport equation for cross-mode coherence was derived using the assumptions of small angle scattering and the Markov approximation, yet while the derivation is formally rigorous, relying on operator methods, it is somewhat opaque with regard to the physical assumptions. Thus we provide here a heuristic derivation of the same equation that, for some, may better elucidate the physical concepts.

Using Eq. (3) the cross-mode coherence equation is

$$
\begin{aligned}
\frac{d \psi_{n} \psi_{p}^{*}}{d r} & =\psi_{p}^{*} \frac{d \psi_{n}}{d r}+\psi_{n} \frac{d \psi_{p}^{*}}{d r} \\
& =-i \sum_{m=1}^{N}\left(\rho_{m n}(r) e^{i l_{m n} r} \psi_{m} \psi_{p}^{*}-\rho_{m p}(r) e^{\left.-i l_{m p}^{*}{ }^{r} \psi_{n} \psi_{m}^{*}\right)} .\right.
\end{aligned}
$$

For small angle scattering the coupling matrix itself is small, so it is useful to solve Eq. (13) by iteration (Sakurai, 1985). Each order of the iterated solution is interpreted physically in terms of multiple scattering, so to second order (second order scattering) the solution is

$$
\begin{aligned}
\psi_{n} \psi_{p}^{*}( & r) \\
= & \psi_{n} \psi_{p}^{*}(0)-\sum_{m=1}^{N} \sum_{q=1}^{N} \int_{0}^{r} d r^{\prime} \int_{0}^{r^{\prime}} d r^{\prime \prime}\left(\psi_{q} \psi_{p}^{*}(0) \rho_{m n}\left(r^{\prime}\right) \rho_{q m}\left(r^{\prime \prime}\right)\right. \\
& \times e^{i\left(l_{m n} r^{\prime}+l_{q m} r^{\prime \prime}\right)}-\psi_{m} \psi_{q}^{*}(0) \rho_{m n}\left(r^{\prime}\right) \rho_{q p}\left(r^{\prime \prime}\right) e^{i\left(l_{m n} r^{\prime}-l_{q p}^{*} r^{\prime \prime}\right)} \\
& -\psi_{q} \psi_{m}^{*}(0) \rho_{m p}\left(r^{\prime}\right) \rho_{q n}\left(r^{\prime \prime}\right) e^{-i\left(l_{m p}^{*} r^{\prime}-l_{q n} r^{\prime \prime}\right)} \\
& \left.+\psi_{n} \psi_{q}^{*}(0) \rho_{m p}\left(r^{\prime}\right) \rho_{q m}\left(r^{\prime \prime}\right) e^{-i\left(l_{m p}^{*} r^{\prime}+l_{q m}^{*} r^{\prime \prime}\right)}\right),
\end{aligned}
$$

where $r$ is the range. The first order terms have been left out of Eq. (14) because they will drop out when the expectation value is taken since $\left\langle\rho_{m n}\right\rangle=0$. Here the objective is to derive an evolution equation for the cross-mode coherence matrix as a function of range which may include many correlation lengths of the random sound-speed structure. We make use of Eq. (14) and conceptually consider the change in the coherence matrix over a distance $r$ when some initial field $\psi_{n} \psi_{p}^{*}(0)$ is incident upon a section of sound-speed fluctuations. The range $r$ is loosely defined such that $r \gg r_{c}$ with $r_{c}$ the largest correlation length of any of the coupling matrix terms. Taking the expectation value of Eq. (14) and making the reasonable assumption that the initial coherence matrix is uncorrelated with the subsequent coupling matrices, the result is

$$
\begin{aligned}
\gamma_{n p}(r)= & \gamma_{n p}(0)-\sum_{m=1}^{N} \sum_{q=1}^{N} \int_{0}^{r} d r^{\prime} \int_{0}^{r^{\prime}} d r^{\prime \prime}\left(\gamma_{q p}(0) \Delta_{m n, q m}(\xi)\right. \\
& \times e^{i\left(l_{m n} r^{\prime}+l_{q m^{\prime \prime}}\right)}-\gamma_{m q}(0) \Delta_{m n, q p}(\xi) e^{i\left(l_{m n} r^{\prime}-l_{q p}^{*} r^{\prime \prime}\right)} \\
& -\gamma_{q m}(0) \Delta_{m p, q n}(\xi) e^{-i\left(l_{m p}^{*} r^{\prime}-l_{q n} r^{\prime \prime}\right)} \\
& \left.+\gamma_{n q}(0) \Delta_{m p, q m}(\xi) e^{-i\left(l_{m p}^{*} r^{\prime}+l_{q m}^{*} r^{\prime \prime}\right)}\right)
\end{aligned}
$$

where $\gamma_{n p}=\left\langle\psi_{n}(r) \psi_{p}^{*}(r)\right\rangle, \Delta_{m n, q p}$ are the correlation functions of the coupling matrices previously discussed, and $\xi=r^{\prime}-r^{\prime \prime}$. Changing the $r^{\prime \prime}$ integration variable to $\xi$, assuming that $r$ $\gg r_{c}$ so that the $\xi$ integration limit can go to infinity, and differentiating with respect to $r$ the final transport equation is

$$
\begin{aligned}
\frac{d \gamma_{n p}(r)}{d r}= & -\sum_{m=1}^{N} \sum_{q=1}^{N}\left(\gamma_{q p}(r) I_{m n, q m} e^{i l_{q n} r}\right. \\
& -\gamma_{m q}(r) I_{m n, q p}^{*} e^{i\left(l_{m n} r-l_{q p}^{*} r\right)} \\
& \left.-\gamma_{q m}(r) I_{m p, q n} e^{-i\left(l_{m p}^{*} r-l_{q n} r\right)}+\gamma_{n q}(r) I_{m p, q m}^{*} e^{-i l_{q p}^{*} r}\right),
\end{aligned}
$$

where the $I_{m n, q m}$ matrices are given by Eq. (10), ${ }^{1}$ Importantly on the right-hand side of Eq. (16) the initial coherence matrices $\gamma_{n p}(0)$ have been replaced with the value at range $r$. This approximation is justified for two reasons. First, considering propagation through multiple correlation lengths the initial field must change with range, and second $\gamma_{n p}(0)$ $\simeq \gamma_{n p}(r)$ because the first correction is at second order. The derivation of this transport equation involves the Markov approximation (Van Kampen, 1981; Creamer, 1996; Henyey and Ewart, 2006) since it is assumed that $r \gg r_{c}$ but in the end $r$ is taken to be infinitesimally small; the coupling matrices are thus assumed to be delta correlated. Also, as previously discussed, the present derivation assumes small-angle scattering because only terms to second order are retained in Eq. (13). The conceptual derivation just described can be obtained more rigorously using operator methods (see Van Kampen, 1981 and Creamer, 1996), and Eq. (16) can also be derived by assuming Gaussian statistics for $\rho_{m n}$ (Morozov and Colosi, 2007).

The right-hand side of this equation has factors that depend on $r$, making numerical or further theoretical progress difficult. The $r$ dependence can be removed by undoing the transformation $\psi_{n}=a_{n} e^{i l_{n} r}$; the result is ${ }^{2}$ 


$$
\begin{aligned}
\frac{d\left\langle a_{n} a_{p}^{*}\right\rangle(r)}{d r}= & i\left(l_{n}-l_{p}^{*}\right)\left\langle a_{n} a_{p}^{*}\right\rangle-\sum_{m=1}^{N} \sum_{q=1}^{N}\left(\left\langle a_{q} a_{p}^{*}\right\rangle I_{m n, q m}\right. \\
& -\left\langle a_{m} a_{q}^{*}\right\rangle I_{m n, q p}^{*}-\left\langle a_{q} a_{m}^{*}\right\rangle I_{m p, q n} \\
& \left.+\left\langle a_{n} a_{q}^{*}\right\rangle I_{m p, q m}^{*}\right) .
\end{aligned}
$$

Equation (17) has been shown to be consistent with the cross-mode coherence equation obtained by Voronovich and Ostashev (2009) when they neglect azimuthal coupling (Voronovich and Ostashev, personal communication). The determination of regimes in which the present $2 \mathrm{D}$ approximation breaks down and a fully three-dimensional 3D treatment is needed is an interesting area of future research.

\section{MODE ENERGY}

An acoustic observable that has received much attention since the seminal paper by Dozier and Tappert (1978a, $1978 b$ ) is the mode energy or $\left\langle\left|a_{n}\right|^{2}\right\rangle$ (Creamer, 1996; Colosi and Flatte, 1996; Tielburger et al., 1997; Wage et al., 2005). For the case of weak attenuation a state of equipartitioning of modal energy is understood to be a modal manifestation of full saturation (Flatté et al., 1979). However, theoretical work to date has not been able to address the influences of the cross-mode coherences on the modal energy evolution. From Eq. (17) the evolution of the mode energy is given by

$$
\begin{aligned}
\frac{d\left\langle\left|a_{n}\right|^{2}\right\rangle}{d r}+2 \alpha_{n}\left\langle\left|a_{n}\right|^{2}\right\rangle= & \sum_{m=1}^{N}\left(\left\langle\left|a_{m}\right|^{2}\right\rangle f_{m n}-\left\langle\left|a_{n}\right|^{2}\right\rangle g_{m n}\right) \\
& -\sum_{m=1}^{N} \sum_{q=1, q \neq n}^{N} 2 \operatorname{Re}\left(\left\langle a_{q} a_{n}^{*}\right\rangle I_{m n, q m}\right) \\
& +\sum_{m=1}^{N} \sum_{q=1, q \neq m}^{N} 2 \operatorname{Re}\left(\left\langle a_{q} a_{m}^{*}\right\rangle I_{m n, q n}\right),
\end{aligned}
$$

where the diagonal contributions (single sum terms) have been separated from the cross-mode coherence contributions (double sums). In this equation the important matrices are

$$
f_{m n}=2 \operatorname{Re}\left(I_{m n, n m}\right)=2 \int_{0}^{\infty} d \xi \Delta_{m n, m n}(\xi) \cos \left(k_{m n} \xi\right) e^{+\alpha_{m n} \xi}
$$

and $g_{m n}=2 \operatorname{Re}\left(I_{m n, m n}\right)$. Because the matrices $f_{m n}$ and $g_{m n}$ are positive definite quantities, whereas the terms $\operatorname{Re}\left(\left\langle a_{q} a_{n}^{*}\right\rangle I_{m n, q m}\right)$ and $\operatorname{Re}\left(\left\langle a_{q} a_{m}^{*}\right\rangle I_{m n, q n}\right)$ are highly oscillatory functions of the indices, the mode energy evolution equations are insensitive to the cross-mode coherences; this fact will be exemplified shortly with a numerical example. Therefore, ignoring the cross-mode coherence terms and assuming that the attenuation is weak enough that the variation in the exponentials in $f_{m n}$ and $g_{m n}$ are weak over the short correlation length of the coupling matrix ${ }^{3}$ (Creamer, 1996), the mode energy equations become

$$
\frac{\partial\left\langle\left|a_{n}\right|^{2}\right\rangle}{\partial r}+2 \alpha_{n}\left\langle\left|a_{n}\right|^{2}\right\rangle=\sum_{m=1}^{N} f_{m n}\left(\left\langle\left|a_{m}\right|^{2}\right\rangle-\left\langle\left|a_{n}\right|^{2}\right\rangle\right) .
$$

In this approximation the matrix $f_{m n}=g_{m n}=2 \pi\langle| \hat{\rho}_{m n}(k$ $\left.\left.=k_{m n}\right)\left.\right|^{2}\right\rangle$ reveals the important resonance condition described by Dozier and Tappert (1978a, 1978b) in which only internal waves whose horizontal wavenumber matches the beat wavenumber $k_{m n}$ contribute to the coupling between modes $m$ and $n$. Here $\left\langle\left|\hat{\rho}_{m n}\left(k=k_{m n}\right)\right|^{2}\right\rangle$ is the wavenumber spectrum of the coupling matrix between modes $n$ and $m$. Equation (20) was obtained by Creamer (1996) and with $\alpha_{n}=0$, Dozier and Tappert's (1978a, 1978b) "master equations" are recovered.

For the deep-water example demonstrated in Fig. 1, a comparison of the mode energies computed by the Dozier Tappert's (1978a, 1978b) equations and the full evolution equations including the coherences is shown in Fig. 2 (see the Appendix for computational and environmental parameters). Here it is seen that aside from some small oscillations the Dozier Tappert's (1978a, 1978b) results closely models the solution to the full equations. In Fig. 2 mode energies in the neighborhood of mode 20 are considered since our initial condition gives mode 20 the largest initial energy. Other calculations that have been done at different frequencies, source depths, and point source initial conditions show the same behavior as in Fig. 2, and therefore the result appears to be quite robust.

\section{A TWO-MODE EXAMPLE}

A two-mode example is useful toward gaining an understanding of the structure of the evolution equations and the relative rates of change in mode energy and coherence as a function of range. Using Eq. (17) and the approximation from Sec. III that the attenuation is weak enough to be ignored in the $I_{m n, p q}$ terms, it is found that several terms simplify to yield the three evolution equations:

$$
\begin{aligned}
\frac{d\left\langle\left|a_{1}\right|^{2}\right\rangle}{d r}+2 \alpha_{1}\left\langle\left|a_{1}\right|^{2}\right\rangle= & 2 \operatorname{Re}\left(I_{12,12}\right)\left(\left\langle\left|a_{2}\right|^{2}\right\rangle-\left\langle\left|a_{1}\right|^{2}\right\rangle\right) \\
& +2\left(I_{21,11}-I_{21,22}\right) \operatorname{Re}\left(\left\langle a_{1} a_{2}^{*}\right\rangle\right),
\end{aligned}
$$

$$
\begin{aligned}
\frac{d\left\langle\left|a_{2}\right|^{2}\right\rangle}{d r}+2 \alpha_{2}\left\langle\left|a_{2}\right|^{2}\right\rangle= & 2 \operatorname{Re}\left(I_{12,12}\right)\left(\left\langle\left|a_{1}\right|^{2}\right\rangle-\left\langle\left|a_{2}\right|^{2}\right\rangle\right) \\
& +2\left(I_{21,22}-I_{21,11}\right) \operatorname{Re}\left(\left\langle a_{1} a_{2}^{*}\right\rangle\right),
\end{aligned}
$$

$$
\begin{aligned}
& \frac{d\left\langle a_{1} a_{2}^{*}\right\rangle}{d r}+i\left(l_{2}^{*}-l_{1}\right)\left\langle a_{1} a_{2}^{*}\right\rangle \\
& =-\left(I_{11,11}+I_{22,22}-2 I_{11,22}+2 I_{12,12}\right)\left\langle a_{1} a_{2}^{*}\right\rangle \\
& \quad+\left(I_{11,21}-I_{22,21}\right)\left\langle\left|a_{1}\right|^{2}\right\rangle+\left(I_{22,21}-I_{11,21}\right)\left\langle\left|a_{2}\right|^{2}\right\rangle .
\end{aligned}
$$

In Eqs. (21) and (22) as previously noted the effect of the coherence should be small because $\operatorname{Re}\left(\left\langle a_{1} a_{2}^{*}\right\rangle\right)$ oscillates. 

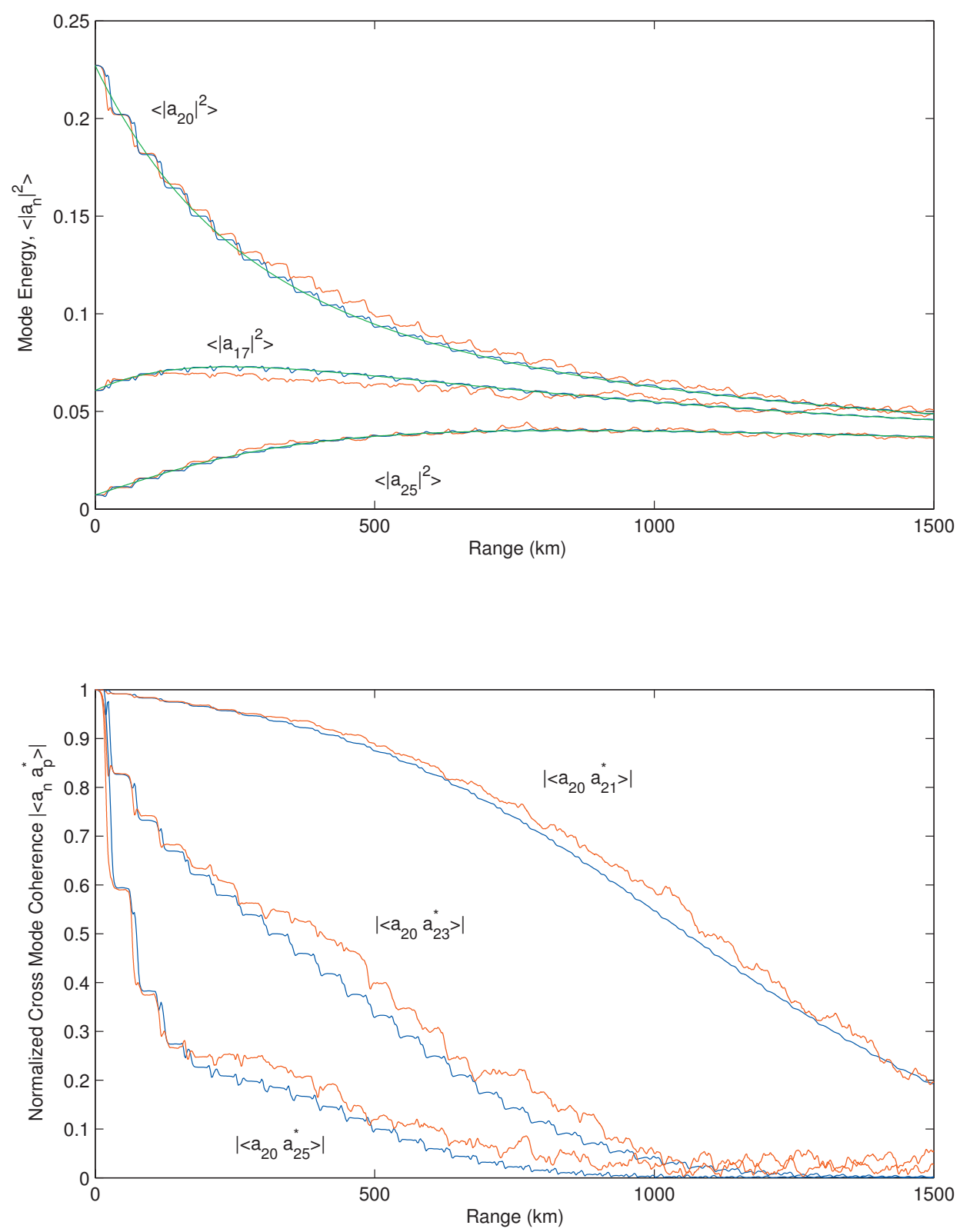

FIG. 2. Simulation and theoretical predictions for mode energy (upper panel) and cross-mode coherence (lower panel) for a few modes. In the upper panel energy for modes 17,20 , and 25 are plotted for the Monte Carlo simulation (red), Dozier-Tappert model [Eq. (20); green], and the full coherence matrix calculation [Eq. (17); blue]. In the lower panel crossmode coherences between mode 20 and modes 21,23 , and 25 are shown. Monte Carlo simulation results are shown in red, while model results from Eq. (17) are shown in blue.
Further it can be noted in this two-mode example that the terms $I_{21,11}-I_{21,22}$ and $I_{11,21}-I_{22,21}$ can also be small if the projection of the sound-speed fluctuations on the two-mode functions [quantified through the functions $G_{m n}(j)$ ] is not a strong function of mode number. If this is the case the last terms in Eqs. (21)-(23) can be ignored, yielding coupled equations for the mode energies and a single equation for the coherence,

$$
\begin{aligned}
& \frac{d\left\langle\left|a_{1}\right|^{2}\right\rangle}{d r}+2 \alpha_{1}\left\langle\left|a_{1}\right|^{2}\right\rangle=2 \operatorname{Re}\left(I_{12,12}\right)\left(\left\langle\left|a_{2}\right|^{2}\right\rangle-\left\langle\left|a_{1}\right|^{2}\right\rangle\right), \\
& \frac{d\left\langle\left|a_{2}\right|^{2}\right\rangle}{d r}+2 \alpha_{2}\left\langle\left|a_{2}\right|^{2}\right\rangle=2 \operatorname{Re}\left(I_{12,12}\right)\left(\left\langle\left|a_{1}\right|^{2}\right\rangle-\left\langle\left|a_{2}\right|^{2}\right\rangle\right), \\
& \frac{d\left\langle a_{1} a_{2}^{*}\right\rangle}{d r}+i\left(l_{2}^{*}-l_{1}\right)\left\langle a_{1} a_{2}^{*}\right\rangle=-\left(I_{11,11}+I_{22,22}-2 I_{11,22}\right. \\
& \left.+2 I_{12,12}\right)\left\langle a_{1} a_{2}^{*}\right\rangle .
\end{aligned}
$$

Here the transfer of energy between modes 1 and 2 is con- trolled by the familiar matrix element $2 \operatorname{Re}\left(I_{12,12}\right)=f_{12}$. Numerical tests not shown here for lack of space reveal that Eqs. (24)-(26) are an excellent approximation to the full equations. Solution to equations like Eqs. (21) and (22) with constant coefficients are easily obtained by eigenvector analysis. Here we are primarily interested in the exponential decay rates given by the eigenvalues which are, $\lambda_{ \pm}=-\left(f_{12}\right.$ $\left.+\alpha_{1}+\alpha_{2}\right) \pm \sqrt{f_{12}^{2}+\left(\alpha_{1}-\alpha_{2}\right)^{2}}$. In the absence of attenuation the eigenvalues are $2 f_{12}$ and zero, so the approach to modal energy equipartition is dictated by $2 f_{12}$. When small attenuation is added to the picture such that $f_{12} \gg \alpha_{1}, \alpha_{2}$, the eigenvalues are slightly modified giving $2 f_{12}+\alpha_{1}+\alpha_{2}$ and $\alpha_{1}+\alpha_{2}$; that is to say, we have the coupling induced rate to equipartition superimposed on the slow overall attenuation decay of the mode amplitudes. This might be the case when the acoustic frequency is high or for deep-water propagation where $\alpha_{n}$ is small. If the attenuation is larger such that $f_{12} \ll \alpha_{1}, \alpha_{2}$ the approach to equipartition is dramatically changed. In this case the eigenvalues are $f_{12}+2 \alpha_{1}$ and $f_{12}+2 \alpha_{2}$, and the at- 
tenuation dominates the exponential decay of the modes; this is the case in shallow water.

For the cross-mode coherence the solution of Eq. (26) is

$$
\left\langle a_{1} a_{2}^{*}\right\rangle(r)=\left\langle a_{1} a_{2}^{*}\right\rangle(0) e^{i\left(l_{1}-l_{2}^{*}\right) r} e^{-\left(I_{11,11}-2 I_{11,22}+I_{22,22}\right) r} e^{-2 I_{12,12} r} .
$$

In the coherence solution attenuation only enters through the first exponential term and results in a slow decay of each of the initial mode amplitudes. The remaining terms are to be physically interpreted as phase randomization terms caused by adiabatic effects and coupling: the adiabatic terms are addressed first. In the adiabatic approximation the coupling matrices are diagonal and thus the cross-mode coherence can be easily written as

$$
\begin{aligned}
\left\langle a_{n} a_{p}^{*}\right\rangle(r)= & \left\langle a_{n} a_{p}^{*}\right\rangle(0) e^{i\left(l_{n}-l_{p}^{*}\right) r} \exp \left(-\frac{1}{2}\left\langle\left(\int_{0}^{r} \rho_{n n}(r) d r\right.\right.\right. \\
& \left.\left.\left.-\int_{0}^{r} \rho_{p p}(r) d r\right)^{2}\right\rangle\right),
\end{aligned}
$$

where it has been assumed here that the coupling matrices are Gaussian random variables. The term in the exponent of Eq. (28) is one-half the adiabatic phase structure function: The phase structure function has a deep connection to coherence (Flatté et al., 1979). In terms of the coherence matrices used in this paper the result is

$$
\begin{gathered}
\frac{1}{2}\left\langle\left(\int_{0}^{r} \rho_{n n}(r) d r-\int_{0}^{r} \rho_{p p}(r) d r\right)^{2}\right\rangle \\
=\left(I_{n n, n n}+I_{p p, p p}-2 I_{n n, p p}\right) r,
\end{gathered}
$$

where it has been assumed that $r \gg r_{c}$. Hence the second exponential term in Eq. (27) is immediately recognized to be the adiabatic contribution resulting from the adiabatic phase structure function.

The last exponential term in Eq. (27) comes from mode coupling and has both real and imaginary parts. Here the coherence decay from mode coupling goes with the rate $2 \operatorname{Re}\left(I_{12,12}\right)=f_{12}$, which is exactly half the rate driving the modes to energy equipartition. If adiabatic effects are weak, then the coherence decay is dominated by coupling and thus mode energy and cross-mode coherence decay at similar rates. In deep-water environments and for frequencies of order tens to hundreds of hertz it has been found that adiabatic effects are indeed weak and coupling is dominant (Dozier and Tappert, 1978a, 1978b; Colosi and Flatté, 1996). A multi-mode numerical example, demonstrating the relative decay rates of mode energy and coherence in a deep-water setting, will be presented in Sec. V. This example is significant since previous work has suggested that generally the coherence decays more rapidly than the modal energies [Dozier and Tappert (1978a, 1978b); Creamer (1996)]: Now this is understood to be true only in a coupling dominated regime. It is important to note that in shallow-water environments it has been shown by Monte Carlo simulation that cross-mode coherences do decay more rapidly than the mode energies (Creamer, 1996). The physical interpretation of this result [confirmed by theoretical evaluation of Eq. (16) for shallow water] is that shallow-water environments have a strong adiabatic component to mode propagation through random linear internal waves. A full treatment of shallowwater issues is beyond the scope of the present paper and will be addressed in subsequent work.

\section{COMPARISONS TO MONTE CARLO SIMULATION}

To exemplify the accuracy of the cross-mode coherence transport equation and to further illustrate some of the concepts previously put forth, comparisons between Monte Carlo simulations of mode propagation through random realizations of internal waves and results from the theory are presented. To not broaden the scope of this paper too much we only present a deep-water setting where attenuation is absent (see the Appendix for details). Following Morozov and Colosi $(2005$, 2007) and as demonstrated in Fig. 1, a $100-\mathrm{Hz}$ directed acoustical beam is considered. For this case the initial mode excitation distribution is centered on mode 20 with a width of roughly \pm 5 ; a total of $N=60$ modes is considered. Figure 2 shows a comparison between mode energy and cross -mode coherence results for the Monte Carlo simulation based on 500 realizations and predictions based on Eq. (17). In Fig. 2 for clarity of presentation only a few mode combinations are displayed in the neighborhood of the mode number 20 , but the results are very similar for other combinations. It must be noted that in the lower panel of Fig. 2 results are presented for the normalized cross-mode coher-

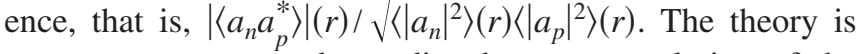
seen to very accurately predict the range evolution of the cross-mode coherence matrix. It should be noted that the decay of the cross-mode coherences by roughly $1500-\mathrm{km}$ range in this example is not inconsistent with basin-scale deep-water observations showing time resolved acoustic wavefronts up to 5000-km range (Worcester et al., 1999). ${ }^{4}$ These wavefronts are due to coherence of the modes across frequency, a problem that will be treated in future work.

The previous comparison is compelling but the most important result is for the real acoustic observable of mean intensity. Figure 3 shows calculations of mean intensity based on the Monte Carlo simulation, and the theory, as well as a calculation of the unperturbed intensity. Example receiver depths are $1.0 \mathrm{~km}$, which is the sound channel axis and $2.2 \mathrm{~km}$, which is in the deep shadow zone of the unperturbed beam. Cylindrical spreading effects are removed from Fig. 3. The Monte Carlo simulation and the theory prediction are seen to be quite different from the unperturbed intensity, demonstrating the well known effect that internal wave can cause a significant mean change in intensity as well as a fluctuation. In particular, for the receiver in the deep shadow zone a difference of $10 \mathrm{~dB}$ or more is evident at long range. Most importantly however is the accuracy of the theory compared to the Monte Carlo calculation; rms differences are less than a decibel, and perhaps primarily due to sampling uncertainty in the Monte Carlo calculation.

\section{SUMMARY AND CONCLUSIONS}

We have presented some further analysis of Creamer's transport equation for the cross-mode coherence matrix 

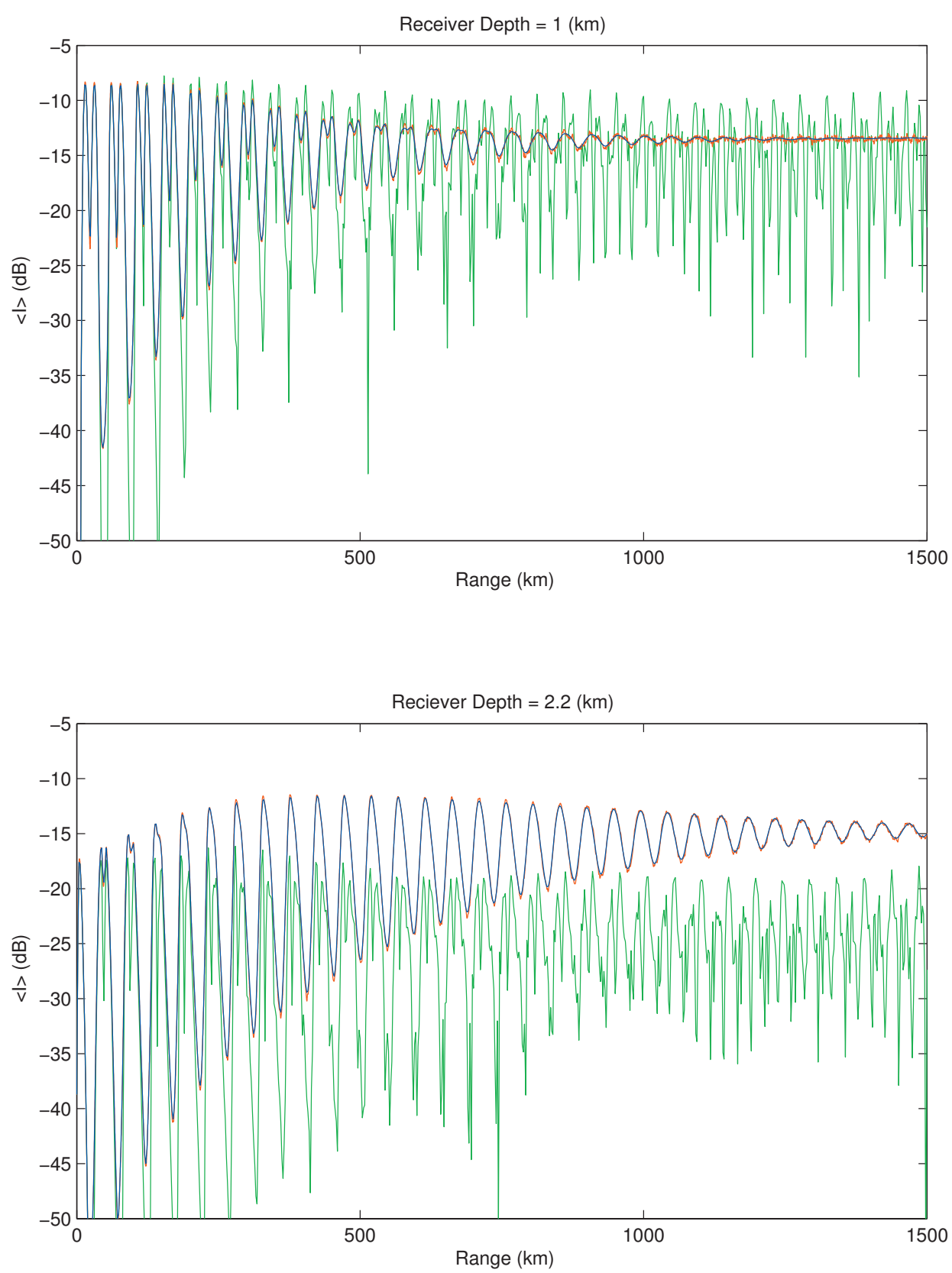

FIG. 3. Mean intensity from the Monte Carlo simulation (red), the model results from Eq. (17) (blue), and the unperturbed intensity $\left(\mu_{0}=0\right.$; green). In each calculation Eq. (2) is utilized but the cylindrical spreading factor is ignored. The upper/lower panels show cases for receiver depths at $1.0 / 2.2 \mathrm{~km}$.
(Creamer, 1996). Three main results are found. First, the diagonal elements of the transport equations which dictate the range evolution of the mode energy are seen to be roughly independent of the off-diagonal elements of the cross-mode coherence matrix. Second, in the deep-water cases addressed here the off-diagonal elements of the cross coherence matrix are seen to evolve in range at similar rates to the diagonal elements or the mode energy. This result comes from a dominance of mode coupling phase randomization effects over those from adiabatic randomization. In shallow-water cases, not treated in detail in this paper but in others (Creamer, 1996) it is found that the opposite is true; cross-mode coherences decay much more rapidly than mode energy because the mode coupling rates are so slow and the adiabatic effect is so strong. Finally, it is shown that Creamer's (1996) transport equation can be used to accurately predict low frequency mean intensity for sound transmission through internal waves in a deep-water environment.
Challenges now are to understand the breakdown of the theory at higher frequency where the coupling gets stronger and to extend the theory to include cross-mode coherence between two different frequencies and thereby treat pulse propagation. The limitations of this $2 \mathrm{D}$ approach compared to the 3D treatment of Voronovich and Ostashev (2009) are also of fundamental interest. In addition the accuracy of the model in shallow-water environments where attenuation effects are important needs to be established. Finally comparisons to observations will provide the ultimate test of the utility of the theory and assumed ocean model as an acoustic prediction tool.

\section{ACKNOWLEDGMENTS}

The authors appreciate many useful discussions with Frank Henyey, Alex Voronovich, Vladimir Ostashev, and members of the North Pacific Acoustic Laboratory group. 
This work was supported by the Office of Naval Research and the Naval Undersea Warfare Center's (NUWC) UnderSea Warfare (USW) chair at the Naval Postgraduate School.

\section{APPENDIX: MONTE CARLO SIMULATION AND MODEL CALCULATIONS}

In this paper, example calculations are presented utilizing a simple deep-water environment, in which attenuation is neglected. A 2D sound-speed field of the form $c(r, z)=\bar{c}(z)$ $+\delta c(r, z)$ is considered, where $\delta c$ is the internal wave perturbation which is small compared to the mean $\bar{c}(z)$. The mean sound-speed profile is modeled using the Munk canonical form (Munk, 1974)

$$
\bar{c}(z)=c_{0}\left[1+\epsilon\left(e^{-2\left(z-z_{a}\right) / B}+2\left(z-z_{a}\right) / B-1\right)\right],
$$

with parameters $c_{0}=1500 \mathrm{~m} / \mathrm{s}$, the sound channel axial depth $z_{a}=1000 \mathrm{~m}, B=1000 \mathrm{~m}$, and $\epsilon=0.005515$. The total water depth is chosen to be $D=4000 \mathrm{~m}$, and the background density is $\rho_{0}=1025 \mathrm{~kg} / \mathrm{m}^{3}$. Internal-wave-induced soundspeed perturbations, $\delta c$, are modeled using the method of Colosi and Brown (1998); however, instead of using the Garrett-Munk spectrum the approximation in Eq. (8) is utilized. In these calculations the buoyancy frequency profile has an exponential form $N(z)=N_{0} e^{-z / B}$, where $B=1000 \mathrm{~m}$ and $N_{0}=5 \mathrm{cph}$. In our numerical calculations a maximum internal wave mode number of 100 is used, and internal waves with horizontal scales from 0.5 to $1600 \mathrm{~km}$ are simulated. Finally, the fractional sound-speed variance is modeled to be

$$
\left\langle\mu^{2}(z)\right\rangle=\left\langle\mu_{0}^{2}\right\rangle\left(N(z) / N_{\text {ref }}\right)^{3},
$$

where $\left\langle\mu_{0}^{2}\right\rangle=6.26 \times 10^{-8}$ and $N_{\text {ref }}=3 \mathrm{cph}$. It should be noted, however, that the actual simulation profile of $\left\langle\mu^{2}(z)\right\rangle$ will be modified from Eq. (A2) near the ocean surface and bottom since the internal-wave vertical modes have a zero displacement boundary condition (see Flatte and Colosi, 2008).

Monte Carlo numerical simulations were carried out with the aforementioned environmental parameters. A $100-\mathrm{Hz}$ acoustical beam is considered [Morozov and Colosi $(2005,2007)]$, where the initial condition is

$$
a_{n}(0)=N_{a}^{-1} \sqrt{k_{0} / \delta} \int_{0}^{D}\left(\phi_{n}(z) / \sqrt{\rho_{0}(z)}\right) e^{-k_{0}^{2}\left(z-z_{s}\right)^{2} / \delta^{2}} d z,
$$

with $\delta=40, z_{s}=2000 \mathrm{~m}$, and $N_{a}$ is a normalization factor such that $\sum_{n=1}^{N}\left\langle\left|a_{n}(0)\right|^{2}\right\rangle=1$. The maximum mode number $N$ is 60 , and the mode number with the largest initial energy is mode 20. The coupled mode equations are solved for random realizations of the internal-wave-induced sound-speed perturbations by transforming Eq. (3) to be expressed in terms of the mode amplitude $a_{n}$ instead of $\psi_{n}$; the coupled equations are then solved using eigenvector techniques, as described in Dozier and Tappert (1978a, 1978b) and Creamer (1996). A total of 500 realizations of $a_{n}(r)$ was computed to obtain ensemble averages of the cross-mode coherence matrix and the mean intensity.

\footnotetext{
${ }^{1}$ Equation (16) is equivalent to Eq. (17) in Creamer (1996).
}

${ }^{2}$ Importantly if attenuation is neglected the resulting symmetry of the matrices means that Eq. (17) conserves energy, that, is $d / d r \sum_{n=1}^{N}\left\langle\left|a_{n}\right|^{2}\right\rangle(r)$ $=0$.

${ }^{3}$ Ignoring the exponentials is a small attenuation, high frequency approximation. In the absence of attenuation there is a coupling resonance condition that selects only the internal-wave wavenumber that matches the beat wavenumber $k_{m n}$. Physically, attenuation broadens the resonance condition so that more internal-wave wavenumbers contribute to the coupling (Colosi, 2008).

${ }^{4}$ The initial condition in this example is clearly different from the point source initial condition in the observations.

Beron-Vera, F. J., Brown, M. G., Colosi, J. A., Virovlyansky, A. L., Zaslavsky, G. M., Tomsovic, S., and Wolfson, M. A. (2003). "Ray dynamics in a long range acoustic propagation experiment," J. Acoust. Soc. Am. 114, 1226-1242.

Colosi, J. A. (2008). "Acoustic mode coupling induced by shallow water nonlinear internal waves: Sensitivity to environmental conditions and space-time scales of internal waves," J. Acoust. Soc. Am. 124, 1452-1464. Colosi, J. A., and Brown, M. G. (1998). "Efficient numerical simulation of stochastic internal wave induced sound speed perturbation fields," J. Acoust. Soc. Am. 103, 2232-2235.

Colosi, J. A., and Flatté, S. M. (1996). "Mode coupling by internal waves for multimegameter acoustic propagation in the ocean," J. Acoust. Soc. Am. 100, 3607-3620.

Colosi, J. A., Flatté, S. M., and Bracher, C. (1994). "Internal wave effects on 1000-km oceanic acoustic pulse propagation: Simulation and comparison to experiment," J. Acoust. Soc. Am. 96, 452-468.

Colosi, J. A., Scheer, E. K., Flatte, S. M., Cornuelle, B. D., Dzieciuch, M. A., Munk, W. H., Worcester, P. F., Howe, B. M., Mercer, J. A., Spindel, R. C., Metzger, K., Birdsall, T. G., and Baggeroer, A. B. (1999). "Comparisons of measured and predicted acoustic fluctuations for a 3250-km propagation experiment in the eastern North Pacific Ocean," J. Acoust. Soc. Am. 105, 3202-3218.

Creamer, D. (1996). "Scintillating shallow water waveguides," J. Acoust. Soc. Am. 99, 2825-2838.

Dozier, L. B. (1983). "A coupled mode model for spatial coherence of bottom-interacting energy," in Proceedings of the Stochastic Modeling Workshop, edited by C. W. Spofford and J. M. Haynes (ARL-University of Texas, Austin TX).

Dozier, L. B., and Tappert, F. D. (1978a). "Statistics of normal-mode amplitudes in a random ocean. I. Theory," J. Acoust. Soc. Am. 63, 353-365. Dozier, L. B., and Tappert, F. D. (1978b). "Statistics of normal-mode amplitudes in a random ocean. II. Computations," J. Acoust. Soc. Am. 64, 533-547.

Dushaw, B. D., Howe, B. M., Mercer, J. A., and Spindel, R. C. (1999). "Multi-megameter range acoustic data obtained by bottom mounted hydrophone arrays for measurement of ocean temperature," IEEE J. Ocean. Eng. 24, 203-215.

Flatté, S. M., and Colosi, J. A. (2008). "Anisotropy of the wavefront distortion for acoustic pulse propagation through ocean sound speed fluctuations: A ray perspective," IEEE J. Ocean. Eng. 33, 477-488.

Flatté, S. M., Dashen, R., Munk, W., Watson, K., and Zachariasen, F. (1979). Sound Transmission Through a Fluctuating Ocean (Cambridge University Press, Cambridge, UK).

Fredricks, A., Colosi, J. A., Lynch, J. F., Gawarkeiwicz, G., Chiu, C. S., and Abbot, P. (2005). "Analysis of multipath scintillations observed during the summer 1996 New England shelfbreak PRIMER study," J. Acoust. Soc. Am. 117, 1038-1057.

Henyey, F., and Ewart, T. E. (2006). "Validity of the Markov approximation in ocean acoustics," J. Acoust. Soc. Am. 119, 220-231.

Morozov, A. K., and Colosi, J. A. (2005). "Entropy and scintillation analysis for acoustical beam propagation through ocean internal waves," J. Acoust. Soc. Am. 117, 1611-1623.

Morozov, A. K., and Colosi, J. A. (2007). "Stochastic differential equation analysis for sound scattering by random internal waves in the ocean," Acoust. Phys. 53, 335-347.

Munk, W. H. (1974). "Sound channel in an exponentially stratified ocean, with application to SFOAR," J. Acoust. Soc. Am. 55, 220-226.

Sakurai, J. J. (1985). Modern Quantum Mechanics (Addison-Westley, Redwood City, CA).

Tielburger, D., Finette, S., and Wolf, S. (1997). "Acoustic propagation through an internal wave field in a shallow water waveguide," J. Acoust. Soc. Am. 101, 789-808. 
Van Kampen, N. G. (1981). Stochastic Processes in Physics and Chemistry (North-Holland, New York).

Van Uffelen, L. J., Worcester, P. F., Dzieciuch, M. A., and Rudnick, D. L. (2009). "The vertical structure of shadow-zone arrivals at long range in the ocean,” J. Acoust. Soc. Am. 125, 3569-3588.

Virovlyanskii, A. L. (1989). "Correlation of modes in a waveguide with large scale random inhomogeneities," Radiophys. Quantum Electron. 32, 619-624.

Virovlyanskii, A. L., Kosternin, A. G., and Malakhov, A. N. (1989). "Mode fluctuations in a canonical underwater channel," Sov. Phys. Acoust. 35 138-142.

Voronovich, A. G., and Ostashev, V. E. (2006). "Low frequency sound scattering by internal waves in the ocean," J. Acoust. Soc. Am. 119, 14061419.

Voronovich, A. G., and Ostashev, V. E. (2009). "Coherence function of a sound field in an oceanic waveguide with horizontally isotropic statistics," J. Acoust. Soc. Am. 125, 99-110.

Wage, K. E., Dzieciuch, M. A., Worcester, P. F., Howe, B. M., and Mercer, J. A., (2005). "Mode coherence at megameter ranges in the North Pacific Ocean," J. Acoust. Soc. Am. 117, 1565-1581.

Worcester, P. F., Cornuelle, B. D., Dzieciuch, M. A., Munk, W. H., Colosi, J. A., Howe, B. M., Mercer, J. A., Spindel, R. C., Metzger, K., Birdsall, T., and Baggeroer, A. (1999). "A test of basin-scale acoustic thermometry using a large-aperture vertical array at $3250-\mathrm{km}$ range in the eastern North Pacific Ocean," J. Acoust. Soc. Am. 105, 3185-3201.

Worcester, P. F., Cornuelle, B. D., Hildebrand, J., Hodgekiss, W., Duda, T., Boyd, J., Howe, B. M., Mercer, J. A., and Spindel, R. C. (1994). "A comparison between measured and predicted broadband acoustic arrival patterns in travel-time depth coordinates at 1000-km range," J. Acoust. Soc. Am. 95, 3118-3128. 